\title{
THEOREMS OF ACCOLA TYPE FOR HIGHER DIMENSIONAL MANIFOLDS
}

\author{
SU-SHING CHEN
}

\begin{abstract}
Two theorems of Accola concerning automorphisms of Riemann surfaces can be extended to higher dimensional manifolds. Formulas are obtained concerning signatures of compact oriented $4 k$-dimensional differentiable manifolds and EulerPoincaré characteristics of compact differentiable manifolds and compact complex manifolds.
\end{abstract}

1. Introduction. In [1], R. D. M. Accola has obtained formulas which relate invariants (such as the genera and the dimensions of spaces of meromorphic functions) of a Riemann surface $X$ and quotients of $X$ by certain subgroups of a finite automorphism group $G$ of $X$. The purpose of this note is to exhibit some generalizations to higher dimensional manifolds. The author would like to thank the referee and Professor Accola for their comments. Applications of our formulas and some related results will be given in a forthcoming note.

2. Differentiable actions of a finite group. We shall generalize the first theorem of Accola in [1] to differentiable actions of finite groups on compact differentiable manifolds. Instead of the RiemannHurwitz formula, we apply a formula of Conner [3] concerning differentiable actions of a finite group $G$ on a compact differentiable manifold $X$, namely, the Euler-Poincaré characteristic of $X / G$ is given by

$$
\chi(X / G)=\sum_{o \in G} \chi\left(F_{o}\right) / n,
$$

where $n$ is the order of $G$ and $F_{g}$ is the set of points in $X$ fixed under the element $g \in G$. It is clear that the first theorem in [1] is a consequence of the following.

Theorem $1 .^{1}$ Let $X$ be a compact differentiable manifold admitting a finite group $G$ of diffeomorphisms. Let $G_{1}, G_{2}, \cdots, G_{s}$ be subgroups of $G$ so that $G=\bigcup_{k=1}^{s} G_{k}$. For indices $1 \leqq i<j<\cdots<k \leqq s$, let $G_{i j} \ldots k$

Received by the editors November 19, 1970 .

AMS 1970 subject classifications. Primary 32C10, 32C35.

Key words and phrases. Differentiable manifold, complex manifold, analytic automorphism, diffeomorphism, complex harmonic form, Euler-Poincaré characteristic.

${ }^{1}$ Since the Conner formula holds for other categories of spaces (see [3]), the same formula can be obtained for these categories of spaces.

Copyright (c) 1971, American Mathematical Society 
$=G_{i} \cap G_{j} \cap \cdots \cap G_{k}$. Let $n, n_{i j \ldots k}$ be the orders of $G, G_{i j \ldots k}$. Then

$$
\begin{aligned}
n \chi(X / G)= & \sum_{1 \leq i \leq s} n_{i} \chi\left(X / G_{i}\right)-\sum_{1 \leq i<j \leq s} n_{i j} \chi\left(X / G_{i j}\right) \\
& +\sum_{1 \leq i<j<k \leq s} n_{i j k} \chi\left(X / G_{i j k}\right)-\cdots \\
& -(-1)^{\bullet} n_{12} \ldots, \downarrow\left(X / G_{12} \ldots, s\right) .
\end{aligned}
$$

PRoof. Formula (1) implies $n_{i j \ldots k} \chi\left(X / G_{i j \ldots k}\right)=\sum_{o \in G_{i j} \ldots k} \chi\left(F_{o}\right)$ for indices $1 \leqq i<j<\cdots<k \leqq s$. Now formula (2) follows from counting elements in $G$ and the fact that

$$
1=\sum_{i} 1-\sum_{i<j} 1+\sum_{i<j<k} 1 \cdots
$$

Corollary. Suppose for $0 \neq i \neq j \neq 0$, it happens that $G_{i} \cap G_{j}=\{1\}$. $G$ is then said to admit a partition. In that case, formula (2) becomes

$$
n \chi(X / G)=\sum_{i=1}^{\infty} n_{i} \chi\left(X / G_{i}\right)+\chi(X)\left[-\left(\begin{array}{l}
s \\
2
\end{array}\right)+\left(\begin{array}{l}
s \\
3
\end{array}\right)-\left(\begin{array}{l}
s \\
4
\end{array}\right)+\cdots\right]
$$

or

$$
n \chi(X / G)=\sum_{i=1}^{\infty} n_{i} \chi\left(X / G_{i}\right)+(1-s) \chi(X)
$$

3. Finite groups of Accola type. Let $G$ be a finite group and $G_{1}, G_{2}, \cdots, G_{s}$ be normal subgroups of $G$. We shall call $G$ an Accola group if for each irreducible complex representation $\pi$ of $G$ there is an $i(1 \leqq i \leqq s)$ so that $G_{i} \subset$ kernel $\pi$. For indices $1 \leqq i<j<\cdots<k \leqq s$, let $G_{i j \cdots k}=G_{i} G_{j} \cdots G_{k}$. For a finite group $G$, we denote by $R(G)$ the set of complex irreducible representations of $G$. Let $A$ be a finite dimensional complex vector space such that $A$ is the representation space of a complex representation $\rho$ of $G$. The Maschke theorem implies that $\rho$ is completely reducible and

$$
A=\sum_{\pi \in \mathcal{R}(G)} \sum_{j=1}^{t_{\pi}}\left(A_{\pi}\right)_{j}
$$

where $G$ acts irreducibly on $\left(A_{\pi}\right)_{j}$ for each $j$ and gives the irreducible representation $\pi$ ( $\pi$ occurs in $\rho t_{\pi}$ times). If $q_{\pi}=t_{\pi}\left(\operatorname{dim}\left(A_{\pi}\right)_{j}\right)$, then

$$
\operatorname{dim} A=\sum_{\pi \in \mathcal{R}(G)} q_{\boldsymbol{x}}
$$

If $N$ is a normal subgroup of $G$, let $\hat{N}=\{\pi \in R(G) \mid T \in N \Rightarrow \pi(T)=1\}$. That is $\hat{N}$ is the set of irreducible representations which include $N$ in 
their kernels. The following two lemmas are included in [1].

LemMa 1. Let $A_{N}$ be the subspace of $A$ which $N$ leaves pointwise fixed, then $\operatorname{dim} A_{N}=\sum_{\pi \in \hat{N}} q_{\pi}$.

LeMma 2. Let $G$ be an Accola group and $A$ be the representation space of a complex representation $\rho$ of $G$. Then

$\operatorname{dim} A=\sum_{1 \leq i \leq s} \operatorname{dim} A_{i}-\sum_{1 \leq i<j \leq s} \operatorname{dim} A_{i j}+\cdots-(-1)^{\bullet} \operatorname{dim} A_{12 \ldots s,}$

where, for indices $1 \leqq i<j<\cdots<k \leqq s, A_{i j \ldots k}=A_{a_{i j} \ldots k}$, the invariant subspace of $A$ under $G_{i j \ldots k}$.

4. The signature formula. Let $X$ be a compact oriented differentiable manifold of dimension $4 k$. The signature of $X$ is defined as the signature of the quadratic form in $H^{2 k}(X, R)$ given by the cup product. Thus $\operatorname{Sign}(X)=p-q$, where $p$ is the number of + signs in a diagonalization of the quadratic form and $q$ is the number of - signs (see [4], [5]). If $G$ is a finite group of orientation preserving diffeomorphisms acting on $X$, then $H^{2 k}(X, R)$ is a $G$-module. An Accola group $G$ of orientation preserving diffeomorphisms is called admissible if $(1 \leqq i<j<\cdots<k \leqq s) X / G_{i j} \ldots k$ and $X / G$ are again oriented manifolds. The action of $G$ on $H^{2 k}(X, R)$ preserves $Q$. We may decompose $H^{2 k}(X, \boldsymbol{R})$ as $H^{2 k}(X, \boldsymbol{R})=H^{+} \oplus H^{-}$, where $H^{+}$and $H^{-}$are $Q$-orthogonal and where $Q$ is positive definite on $H^{+}$and negative definite on $H^{-}$and $T\left(H^{+}\right)=H^{+}, T\left(H^{-}\right)=H^{-}$for $T \in G$. Thus, $\operatorname{Sign}(X)=$ $\operatorname{dim} H^{+}-\operatorname{dim} H^{-}$.

THEOREM 2. Let $G$ be an admissible Accola group of orientation preserving diffeomorphisms of a compact oriented differentiable manifold $X$ of dimension $4 k$. Then

$$
\begin{aligned}
\operatorname{Sign}(X)=\sum_{1 \leq i \leq 8} \operatorname{Sign}\left(X / G_{i}\right)-\sum_{1 \leq i<j \leq 8} \operatorname{Sign}\left(X / G_{i j}\right)+\cdots \\
-(-1) \cdot \operatorname{Sign}\left(X / G_{12} \ldots,\right) .
\end{aligned}
$$

Proof. Lemma 2 implies that for $G$-invariant vector spaces $H^{+}$ and $H^{-}$,

$$
\begin{aligned}
& \operatorname{dim} H^{+}= \sum_{1 \leq i \leq e} \operatorname{dim} H_{i}^{+}-\sum_{1 \leq i<j \leq s} \operatorname{dim} H_{i j}^{+}+\cdots \\
&-(-1)^{\bullet} \operatorname{dim} H_{12}^{+} \ldots, \\
& \operatorname{dim} H^{-}=\sum_{1 \leq i \leq \&} \operatorname{dim} H_{i}^{-}-\sum_{1 \leq i<j \leq s} \operatorname{dim} H_{i j}^{-}+\cdots \\
&-(-1)^{\bullet} \operatorname{dim} H_{12}^{-} \ldots . .
\end{aligned}
$$


Subtracting (5) from (4), we get (3).

The above theorem is not exactly a generalization of Accola's second theorem, because the signature of a Riemann surface (any manifold of dimension $\neq 4 k$ ) is defined to be zero.

5. The Euler-Poincaré characteristic formula. Let $X$ be a compact complex manifold. We denote by $H^{p, q}(X)$ the finite dimensional vector space of complex harmonic forms of type $(p, q)$ on $X$ and denote by $h^{p, q}(X)$ the dimension of $H^{p, q}(X)$. The Euler-Poincaré characteristic $\chi(X)$ of $X$ is, according to [4],

$$
\chi(X)=\sum_{p, q}(-1)^{p+q} h^{p, q}(X) .
$$

Suppose that $X$ admits a finite group $G$ of analytic automorphisms. In general $X / G$ is not necessarily a manifold (see [2], [6]). We call an Accola group $G$ of analy tic automorphisms admissible if $X / G_{i j} \ldots k$ $(1 \leqq i<j<\cdots<k \leqq s)$ and $X / G$ are manifolds. The vector space $H^{p, q}(X)$ is invariant under $G$ and hence is a representation space of $G$ canonically.

TheOREM 3. Let $G$ be an admissible Accola group of analytic automorphisms of a compact complex manifold $X$. Then

$$
\begin{aligned}
h^{p, q}(X)=\sum_{1 \leq i \leqq s} h^{p, q}\left(X / G_{i}\right)-\sum_{1 \leq i<j \leqq s} h^{p, q}\left(X / G_{i j}\right)+\cdots \\
-(-1)^{s} h^{p, q}\left(X / G_{12} \ldots s\right) .
\end{aligned}
$$

Proof. This follows from Lemma 2.

Theorem 4. Let $G$ be an admissible Accola group of analytic automorphisms of a compact complex manifold $X$. Then

$$
\chi(X)=\sum_{1 \leqq i \leqq s} \chi\left(X / G_{i}\right)-\sum_{1 \leqq i<j \leqq s} \chi\left(X / G_{i j}\right)+\cdots-(-1)^{s} \chi\left(X / G_{12 \ldots s}\right) .
$$

Proof. This follows from

$$
\chi\left(X / G_{i i \ldots k}\right)=\sum_{p, q}(-1)^{p+q} h^{p, q}\left(X / G_{i j \ldots k}\right)
$$

for indices $1 \leqq i<j<\cdots<k \leqq s$.

Theorem 4 is a generalization of the second theorem of Accola. It would be interesting to see whether this theorem is still true for nonadmissible groups.

\section{REFERENCES}

1. R. D. M. Accola, Two theorems on Riemann surfaces with noncyclic automorphism groups, Proc. Amer. Math. Soc. 25 (1970), 598-602. MR 41 \#3747. 
2. W. L. Baily, Jr., On the imbedding of $V$-manifolds in projective space, Amer. J. Math. 79 (1957), 403-430. MR 20 \#6538.

3. P. E. Conner, Concerning the action of a finite group, Proc. Nat. Acad. Sci. U.S.A. 42 (1956), 349-351. MR 18, 61.

4. F. Hirzebruch, Topological methods in algebraic geometry, 3rd ed., Die Grundlehren der math. Wissenschaften, Band 131, Springer-Verlag, Berlin and New York, 1966. MR 34 \#2573.

5. - The signature of ramified coverings, (Papers in honor of K. Kodaira) Global Analysis (Princeton, 1969), Univ. of Tokyo Press, Tokyo, 1969, pp. 253-265. MR 41 \#2707.

6. I. Satake, On a generalization of the notion of manifold, Proc. Nat. Acad. Sci. U.S.A. 42 (1956), 359-363. MR 18, 144.

University of Florida, Gainesville, Florida 32601 